\title{
Expert opinion regarding the prevalence, health risks, obesogenic environment determinants, and public health strategies on obesity. What are we still doing wrong?
}

\author{
ONISEI Tatiana ${ }^{2}$, POP Anca Lucia ${ }^{*}$, IONITA Ana Corina ${ }^{1}$ MOROSAN Elena ${ }^{1}$, \\ UDEANU Denisa ${ }^{1}$, MITITELU Magdalena ${ }^{1}$, VARLAS Valentin ${ }^{1}$, NASUI Bogdana \\ Adriana $^{3}$, ZETU Cornelia ${ }^{4}$, LUPULIASA Dumitru ${ }^{1}$, DRAGANESCU Doina ${ }^{1}$ \\ 'University of Medicine and Pharmacy" Carol Davila" Bucharest (ROMANIA) \\ ${ }^{2}$ National Institute R\&D for Food Bioresources, National Service for Medicinal, Aromatic Plants and Bee Products, 6 Dinu \\ Vintila Street, 2nd District, Bucharest, Romania, Tel/Fax: +40212109128; +40212113639 \\ ${ }^{3}$ University of Medicine and Pharmacy, Cluj-Napoca, Department of Community Health \\ ${ }^{4}$ National Institute of Diabetes, Nutrition and Metabolic Diseases "Prof. Dr. N. Paulescu” Romania \\ Corresponding author: ancapop@hotmail.com
}

\begin{abstract}
The overweight and obesity, as well as their related non-communicable diseases (NCD's) are preventable through lifestyle changes targeted in public health actions - but unfortunately, with little or no success until now. In the present work, we analyzed the actual essential studies in the field - in order to provide and recommend updated strategies to target the public health objectives efficiently. We identified four main topics of high importance in the current approach towards obesity: (1) the increasing prevalence and multiple health consequences (2) current public health $(\mathrm{PH})$ strategies for risk factor reduction and obesity prevention (3) the influence of the obesogenic environment on individual behavior (4) recent data on weight loss and weight loss maintenance programs (5) existence of a new obesity entity - normal weight obesity to be targeted as a pre-morbid state for non-communicable diseases (NCDs). A new approach is needed towards the (1) causative factors; (2) public health measures addressed precisely to the remarkable regional differences in obesity prevalence and trends derived from the ethio-pathogenic factors and $\mathrm{PH}$ recommendations - most of them related to nutrition patterns and food quality - all together with lifestyle and environment measures.
\end{abstract}

Keywords: obesity, overweight, public health strategies, NCD, non-communicable diseases, WHO

\section{Introduction}

NCDs threaten is enlisted on WHO 2030 Agenda for Sustainable Development that includes a target of reducing premature deaths from NCDs by one-third by 2030. Metabolic risk factors contribute to four critical metabolic changes that increase the risk of NCDs: (1) raised blood pressure (2) overweight/obesity (3) hyperglycemia and (4) hyperlipidemia. Detecting, screening and treating these diseases is critical to accomplish the WHO objectives by a integrated approach: including health, education, agriculture, finance - targeted to the modifiable factors that are on the core of the NCD's: tobacco use, physical inactivity, unhealthy diet and the harmful use of alcohol [1,2].

Overweight is charactersed by atipical or excessive body fat accumulation that may impair health, and obesity have risen dramatically among children and adolescents aged 5-19 from just $4 \%$ in 1975 to over $18 \%$ in 2016. Actually, in 2016, $18 \%$ of girls and $19 \%$ of boys were overweight, while $6 \%$ of girls and $8 \%$ of boys were obese (WHO, 2020). Recent researches show that the most dramatic increases in obesity are in developing countries. [3]. In Europe, the overweight rate was $63 \%$ (men) and $54.3 \%$ (women) in 2016, while the obesity rate was $21.9 \%$ (men) and $24.5 \%$ (women). In Romania, $46,4 \%$ of the adult population is overweight, 
while the rate of obese people is $19,4 \%$. The overweight and obesity and the related noncommunicable diseases are mostly preventable but, unfortunately, with no success, despite the public health measures and enormous actual level of knowledge. So, the continuous screening of the databases is of crucial importance in order to identify, correlate, and apply an integrated approach by healthcare specialists. A new tactic is needed to targeted to the (1) causative factors; (2) public health measures derived from them and recommendations - most of them related to nutrition patterns and food quality - all together with lifestyle and environment measures. The objective of this work is to analyze the most important and recent studies in the field in order to provide and recommend updated strategies to target public health objectives efficiently.

\section{Materials and methods}

In the present study we scanned the recent literature and reviewed our experience regarding the prevalence of obesity and health outcomes due to this morbidity in the context of multiple public health measures to limit the continuous increase of the obesity worldwide, we analyzed the role pro-overfeeding actions in the environment acting in opposition with the public health measures and reviewed the recent data on weight loss and weight loss maintenance programs. Data selection: we screened and synthesized the literature on the obesity topic and current barriers in reducing the prevalence, morbidity, and mortality due to obesity during the last five years but also notable references from previous years. Data sources: Electronic databases (PubMed, Data Citation Index, Current Contents Connect, Cross Ref, Scopus, Web of Science, Google Scholar and Medline) we systematically searched for studies using the terms and categories: "obesity", "obesity epidemic" "overweigh"\& comorbidities" "childhood obesity", "obesity and nutritional intervention", "obesity and public health actions" „child obesity", „obesity prevention programs", "normal weight obesity". The search withdraw over 600.000 results on Google Scholar, over 125,483 results on Web of Science, 124.199 on Medline ${ }^{\circledR}$, with a media of over 30.000 articles during the last five years, 6575 on Data Citation Index, Current Contents Connect - 87,129 articles and reviews

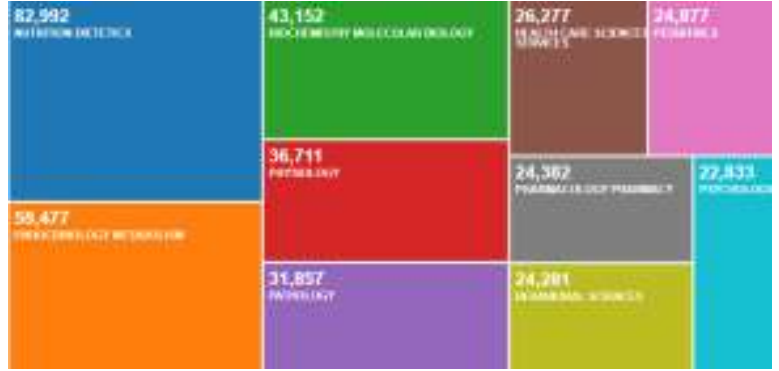

A

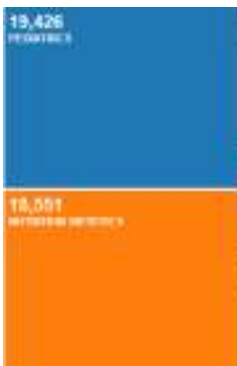

$\mathrm{B}$

Figure 1 Treemap of data results on the search term „obesity” (A) and „child obesity” (B) performed on the Web of Science Databases on a period of five years

From the screened data we selected twelve main topics linked with obesity regarding the obesity prevalence and health consequences, the socio-economic status and obesity, level of education, race and ethnicity, current public health strategies for risk factor reduction and obesity prevention, multifactorial determination of individual behavior in the obesogenic environment, addictive diets and palatability, neuro-endocrine behavioral factors, normal weight obesity, point-of-choice nutrition information, per capita Kcal/day provision in different communities, weight loss nutrition programs.

\section{Results}

The obesity prevalence and health consequences. According to WHO, overweight and obesity are defined as abnormal or excessive body fat accumulation that may impair health 
(WHO, 2020). BMI is a easy index of weight-for-height commonly used to classify overweight $(\mathrm{BMI}>=25)$ and obesity in adults $(\mathrm{BMI}>=30)[4]$.

However, the use of BMI does not individualise the weight associated with muscle and weight associated with fat, so the determination of visceral-abdominal or central fat accumulation is better than BMI to highlight changes in risk factors for cardiovascular diseases and other forms of chronic diseases [5]. Numerous studies have compared the accuracy of various anthropometric indices for assessing obesity and predicting obesity-related health risks, including BMI (James, 2005) [6], waist-to-hip ratio (WHR), waist circumference (WC), and waist-to-height ratio (WHtR) [7]. WHR is a simple and good predictor of health risk, a high WHR (>1.0 in men and >0.85 in women) indicates viscero-abdominal fat accumulation [8]; so a classification of the health risk based on WC is suggested to be more useful for health assessment than either BMI or WHR [9].

In the United States, a higher rate of obesity was registered among adults aged 40-59 as compared to adults aged 20-39, both in men and women [9]. Overall, about one in 3 American adults is obese, followed by Mexicans (30\% rate of obesity in the adult population), New Zealanders (26.5\%), Australians (24.6\%), and Canadians (24.2 percent), where 1 in 4 adult people is obese [1]. In European countries, the rate for overweight was in 2016, about 63\% among men and 54.3\% among women, respectively, while the obesity rate was $21.9 \%$ among men and $24.5 \%$ among women. National-level data for 2016 showed that, in most countries in the E.U. Region, the overweight was more prevalent among men, while obesity was more prevalent among women (WHO, 2018). In Romania, a survey conducted by the National Institute of Statistics, in 2014, showed $46,4 \%$ of the adult population was overweight, while the rate of obese people was $19,4 \%$. A higher percentage of obese women were registered, which could be explained by their sedentary activities as compared with men, which are predominantly overweight. Interestingly, in Romania, obesity has been more common in less educated women, while in men, obesity was observed in those with a high education level.

Socio-economic status (SES) and obesity. overweight and obesity are now on the rise in low- and middle-income countries, due to the increased accesibility of food and a potential decrease in physical activity; nevertheless it was once considered a high-income country problem, particularly in urban settings; Another factor that has been less discussed in previous studies is the lack of self-esteem among low SES people who adopt less positive attitudes towards physical activity [10].

The level of education. Ziraba (2009) showed that in Africa, with increasing urbanization, poverty and social exclusion are liincreasing the risks of developing a chronic disease. The prevalence of overweight and obesity is up to $46 \%$ among non-educated women, while among educated women at the level of secondary school or higher dropped by about $10 \%$ [11]. Similar results have been reported for 2016-2018 in USA - adults with a basic school degree or equivalent had $35.0 \%$ self-reported obesity, high school graduates $(33.1 \%)$, adults with some college (33.0\%) and college graduates (24.7\%) [12]

Race and ethnicity. There are also notable differences by race and ethnicity. A metaanalysis among different ethnic groups showed that - for the same BMI - body fat percentage was 3-5\% higher in Asian populations than Caucasian populations [13]. Combining USA data from 2016 through 2018 highlighted non-Hispanic blacks had the highest prevalence of selfreported obesity (39.1\%), followed by Hispanics (33.3\%) and non-Hispanic whites (29.3\%).

Normal weight obesity. A new guy in town. An individual with a standard body mass index but a high percentage of body fat is an average weight obese (NWO) measured with dualenergy X-ray absorptiometry (DXA) but also ultrasound A techniques [14] with a cutoff point for diagnosis $30 \%$ body fat in Europeans and in recent large scale studies $23.1 \%$ for men and $33.3 \%$ for women [15]; $\geq 23.5 \%$ in men; $\geq 29.2 \%$ in women in Koreans [16, 17]. The situation is gaining attention since due to connection with pro-oxidative effects and systemic low-grade 
chronic subclinical (general and vascular) inflammation, metabolic dysregulation [18, 19, 20] dyslipidemia, insulin resistance [21], changes in blood pressure. An increase in body fat percentage is linked to sedentary behavior and poor eating habits; the affected individuals are seldom identified through routine healthcare needing more than BMI status making mandatory the body composition screening in normal BMI subjects in order to identify people at a high risk of cardiometabolic abnormalities [22].

Current public health strategies for risk factor reduction and obesity prevention. A proposed framework by Sacks (2009) suggests that policy actions to the development and implementation of effective public health strategies to obesity prevention should (1) target the food environments, the physical activity environments and the broader socio-economic environments; (2) directly influence behavior, aiming at improving eating and physical activity behaviors; and (3) support health services and clinical interventions. These areas include fiscal food policies, mandatory nutrition panels on the formulation and reformulation of manufactured foods, implementation of food and nutrition labeling, and restricting marketing and advertising bans of unhealthy foods [23]. The primary aim of the EU (European Union) strategy is to fight against the obesogenic environment - promoting weight gain - and helps citizens reduce high-risk behaviors, such as low nutritional value food and lack of exercise that lead to overweight and obesity. White paper (2007) sets out to achieve this by focusing on actions (information campaigns) that enable consumers to make informed choices to ensure that healthy options are available (e.g. in supermarkets and canteens); encourage the food industry (including retailers) to adjust the salt, sugar, and fats in its product recipes and target responsible marketing to promote healthier options (affordable and available for all population groups, especially children); employers could encourage healthy lifestyles (e.g. "active transport and commuting", walking, cycling or using public transport); motivate people to undertake the regular physical activity (making it more accessible) by stressing the health benefits.

Addictive diets and palatability. Aiming to understand how the pleasure of food affects our brain and behavior, Lee and Dixon have examined the neurobiological and phenotypic similarities and differences between the hedonic pathway triggered by food compared with other addictive substances [24]. The biochemical properties within certain common foods have the potential to cause an addictive process, leading to a typical range of addiction-related problematic behaviors that in some individuals are sufficient to cause clinically significant impairment or distress [25]. The "non-homeostatic" or "hedonic" eating refers to food intake that is not regulated by metabolic feedback and is related to cognitive, reward, and emotional factors [26].

Neuro-endocrine behavioral factors. Individuals vary in their responses to the same stimuli, environmental cues, or signals of hunger and satiety. Once the food is consumed, the motivation to eat is rduced by the processes of satiation and satiety, retrieving episodic feedback from hormonal signals in the gastrointestinal tract and tonic feedback from leptin and insulin, secreted proportionally to the fat mass. Thus, it is proposed that the net effect of these stimulatory and inhibitory signals has a determining influence on eating behaviors like meal size and meal frequency [27]. The development of typical overeating behavior is rooted in the family environment and being internalized, stress-related alleviation-reward systems - are resistant to different nutritional and medical therapies [28] imposing the need for change in the parental attitudes and feeding environment of their child - generating engagement in overeating in response to stress eventually. The nutritional assistance/parenting programs of the families have to be strongly connected with the mandatory psychological assistance [29].

Weight loss and weight loss maintenance programs. Obesity prevention and treatment frequently fail in the long term for the majority of at risk people (for example, behavioral interventions aiming at reducing energy intake and increasing energy expenditure) or are not 
available or suitable (bariatric surgery) [30]. The most effective campaigns are targeting the motivational level of people, shaping people's choices towards the healthier foods and regular physical activity the most comfortable choice - provided by supportive environments and communities together with an increased availability and price of healthy food choices, quality of food, portion sizes, within-outlet promotions, and point-of-choice nutrition information [31].

Point-of-choice nutrition information. Per capita Kcal/day provision. The food industry tends to act opportunistically in the interests of maximizing profits, aim incongruent with the public health efforts for obesity control. The food market in the United States, for example, provides about $3900 \mathrm{kcal}$ per capita each day, roughly twice the population's energy needs [32]. To expand profits in this environment, food companies' strategies promote larger portions, frequent snacking, use of sweets, soft drinks, snacks, and fast food on daily basis. Although the majority agreed that some types of foods are addictive, there was very little support for increasing taxes on these obesogenic foods [33].

Interventions in worksites and through clinical and public health programs. Stress reduction may also be an essential component of weight-loss interventions in worksites and in clinical and public health programs. A mindfulness-based intervention may be effective in reducing stress and improving stress-related overeating. Mindfulness training would enhance awareness of and responsiveness to bodily sensations and reduce psychological distress, emotional eating, and cortisol secretion; may support weight maintenance efforts, and actual weight loss might occur for those participants who eat a high proportion of meals mindfully.

\section{Conclusions}

The first-line treatment of obesity is dietary management combined with behavior modification; secondarily, increased physical activity - safe, efficacious, healthy, and nutritionally adequate, culturally acceptable, and economically affordable diet, without severe restrictions or nutritional exaggerations and should ensure long-term compliance and maintenance of weight loss. Effective public health strategies for obesity prevention should (1) target the food environments, the physical activity environments, the broader socioeconomic environments with intervention at the obesogenic marketing attitudes level; (2) directly influence individual behavior, aiming at improving eating and physical activity behaviors, supporting the sports environments at all age levels, good psychological training and support - and (3) support health services and clinical/nutritional interventions in worksites, through clinical and public health programs, founded on early nutritional education in school curricula and in parents groups. Parenting programs in order to change the parental attitudes and feeding environment of their child, the future adult would be The screening of the new type of normal body weight obesity (NWO) is mandatory for children and adults in order to timely foresee and act to prevent BMI obesity and NCD's.

\section{REFERENCES}

1. Dietz, W., \& Santos-Burgoa, C. (2020). Obesity and its Implications for COVID-19 Mortality. Obesity.

2. GBD 2015 Risk Factors Collaborators. Global, regional, and national comparative risk assessment of 79 behavioural, environmental and occupational, and metabolic risks or clusters of risks, 1990-2015: a systematic analysis for the Global Burden of Disease Study 2015. Lancet, 2016; 388(10053):1659-1724

3. Caballero, B. (2007). The global epidemic of obesity: an overview. Epidemiologic reviews, 29(1), 1-5.

4. Romero-Corral, A., Somers, V. K., Sierra-Johnson, J., Thomas, R. J., Collazo-Clavell, M. L., Korinek, J. E. C., \& Lopez-Jimenez, F. (2008). Accuracy of body mass index in diagnosing obesity in the adult general population. International journal of obesity, 32(6), 959-966.

5. Fabbrini, E., Magkos, F., Mohammed, B. S., Pietka, T., Abumrad, N. A., Patterson, B. W., ... \& Klein, S. (2009). Intrahepatic fat, not visceral fat, is linked with metabolic complications of obesity. Proceedings of the National Academy of Sciences, 106(36), 15430-15435.

6. James, J., \& Kerr, D. (2005). Prevention of childhood obesity by reducing soft drinks. International journal of obesity, 29(2), S54-S57. 
7. McAllister, E. J., Dhurandhar, N. V., Keith, S. W., Aronne, L. J., Barger, J., Baskin, M. \& Elobeid, M. (2009). Ten putative contributors to the obesity epidemic. Critical reviews in food science and nutrition, 49(10), 868-913.

8. Lean, M. E. J. (2000). Pathophysiology of obesity. Proceedings of the Nutrition Society, 59(3), 331-336.

9. Després, J. P. (2001). Health consequences of visceral obesity. Annals of medicine, 33(8), 534-541.

10. Back, J. H., \& Lee, Y. (2011). Gender differences in the association between socio-economic status (SES) and depressive symptoms in older adults. Archives of gerontology and geriatrics, 52(3), e140-e144.

11.Ziraba, A. K., Fotso, J. C., \& Ochako, R. (2009). Overweight and obesity in urban Africa: a problem of the rich or the poor?. BMC public health, 9(1), 465.

12. Prevalence of Self -Reported Obesity Among U.S. Adults by Race/Ethnicity, State and Territory, BRFSS, 2016-2018.

13. Deurenberg, P., Deurenberg-Yap, M., \& Guricci, S. (2002). Asians are different from Caucasians and from each other in their body mass index/body fat per cent relationship. Obesity reviews, 3(3), 141-146.

14. Miclos-Balica M. \& al. (2020) ROMANIAN J. BIOPHYS., Vol. 30, No. 3, pp. 1-14

15. Romero-Corral, A., Somers, V. K., Sierra-Johnson, J., Korenfeld, Y., Boarin, S., Korinek, J., ... \& LopezJimenez, F. (2010). Normal weight obesity: a risk factor for cardiometabolic dysregulation and cardiovascular mortality. European heart journal, 31(6), 737-746.

16. Kim, S., Kyung, C., Park, J. S., Lee, S. P., Kim, H. K., Ahn, C. \& Kang, S. (2015). Normal-weight obesity is associated with increased risk of subclinical atherosclerosis. Cardiovascular Diabetology,14(1), 58.

17. WHO, CONSULTATION, Obesity: Preventing and Managing the Global Epidemic, World Health Organization, Geneva, Switzerland, 2000.

18. Franco, L. P., Morais, C. C., \& Cominetti, C. (2016). Normal-weight obesity syndrome: diagnosis, prevalence, and clinical implications. Nutrition reviews, 74(9), 558-570.

19. Lacatusu, I., Badea, N., Udeanu, D., Coc, L., Pop, A., Negut, C. C. \& Meghea, A. (2019). Improved antiobesity effect of herbal active and endogenous lipids co-loaded lipid nanocarriers: Preparation, in vitro and in vivo evaluation, in vitro and in vivo evaluation, Materials Science \& Engineering C, 99, pp.12-24

20. Kang, S., Kyung, C., Park, J. S., Kim, S., Lee, S. P., Kim, M. K., .. \& Ahn, C. W. (2014). Subclinical vascular inflammation in subjects with normal weight obesity and its association with body fat: an $18 \mathrm{~F}$ FDG-PET/CT study. Cardiovascular diabetology, 13(1), 70.

21. Leți, M.M., Bodnărescu-Cobanoglu M., Dobrescu I., Pop A. L., Popa D., Rosu I. A., Iorgut C., Zetu C. (2018) The Prevalence and Characteristics of Depression in Diabetes Mellitus Patients. Is Routine Screening Of Depression Necessary In The Diabetes Patients? Proceedings of 4 th International Conference on Interdisciplinary Management of Diabetes Mellitus and its Complications INTERDIAB, Filodiritto Editore - Proceedings, pp. -pp. 111-112; 2018

22. García-Hermoso, A., Agostinis-Sobrinho, C., Camargo-Villalba, G. E., González-Jiménez, N. M., Izquierdo, M., Correa-Bautista, J. E., \& Ramírez-Vélez, R. (2020). Normal-Weight Obesity Is Associated with Poorer Cardiometabolic Profile and Lower Physical Fitness Levels in Children and Adolescents. Nutrients, 12(4), 1171.

23. Chan, R. S., \& Woo, J. (2010). Prevention of overweight and obesity: how effective is the current public health approach. International journal of environmental research and public health, 7(3), 765-783.

24. Lee, P. C., \& Dixon, J. (2017). Pharmacotherapy for obesity. Australian family physician, 46(7), 472.

25. Blundell, J., Finlayson, G., Axelsen, M., Flint, A., Gibbons, C., Kvist, T., \& Hjerpsted, J. B. (2017). Effects of once-weekly semaglutide on appetite, energy intake, control of eating, food preference and body weight in subjects with obesity. Diabetes, Obesity and Metabolism, 19(9), 1242-1251.

26. Berthoud, H. R., Lenard, N. R., \& Shin, A. C. (2011). Food reward, hyperphagia, and obesity. American Journal of Physiology-Regulatory, Integrative and Comparative Physiology, 300(6), R1266-R1277.

27. Dalton, M., Finlayson, G., Esdaile, E., \& King, N. (2013). Appetite, satiety, and food reward in obese individuals: a behavioral phenotype approach. Current Nutrition Reports, 2(4), 207-215.

28. Smith, A. D., Sanchez, N., Reynolds, C., Casamassima, M., Verros, M., Annameier, S. K., ... \& Shomaker, L. B. (2020). Associations of parental feeding practices and food reward responsiveness with adolescent stress-eating. Appetite, 104715.

29. Vilaia, D. Parenting \& Family Coach, http://myvolunteercoach.com/parenting/

30. Blüher, M. (2019). Obesity: global epidemiology and pathogenesis. Nature Reviews Endocrinology, 15(5), 288.

31. Sallis, J. F., \& Glanz, K. (2009). Physical activity and food environments: solutions to the obesity epidemic. The Milbank Quarterly, 87(1), 123-154.

32. Ludwig D.S., Nestle M., Can the Food Industry Play a Constructive Role in the Obesity Epidemic?, 2008, JAMA, Vol. 300, Issue 15, Pages 1808-1811

33. Lee P.C., Dixon, J.B., Food for Thought: Reward Mechanisms and Hedonic Overeating in Obesity, 2017 , Current Obesity Reports, Vol. 6, Issue 4, pp. 353-361 